\title{
Biologically-inspired adaptive routing protocol with stochastic route exploration
}

\author{
Tomohiro Nakao, Jun-nosuke Teramae and Naoki Wakamiya \\ Graduate School of Information Science and Technology, Osaka University \\ 1-5 Yamadaoka, Suita, Osaka 565-0871, Japan \\ [ t-nakao,teramae,wakamiya ] @ist.osaka-u.ac.jp
}

\begin{abstract}
Rapid increase in amount of traffic and the number of users of information communication networks requires adaptive routing protocols that can properly respond to unexpected change of communication environment such as rapid and large fluctuation of traffic. While distributed routing protocols that use only local state of the network have been expected to suitable for adaptive routing on large scale network, the lack of global information of the network often makes it difficult to promptly respond to traffic changes of the network when it occurs at out of the local scope. In this paper, based on the biologically-inspired attractor selection model, we propose a distributed routing protocol with active and stochastic route exploration. Acquiring current state of the network beyond its local scope by utilizing stochastic nature of the protocol, the routing protocol can efficiently respond to rapid change of traffic demand on the network. In order to avoid destabilization of routings due to the exploration, we introduce a short-term memory term to the governing equation of the protocol. We also confirm that the protocol successfully balances rapid exploration with stable routing owing to the memory term by numerical simulations.
\end{abstract}

\section{Categories and Subject Descriptors}

C.2.2 [Network Protocols]: routing protocols

\section{Keywords}

distributed routing, Attractor Selection, stochastic exploration, a short-term memory

\section{INTRODUCTION}

Due to rapid increase of the number and diversity of users of the Internet, temporal variation of traffic on the Internet becomes rapid and large recently. While periodic variations of traffic such as daily fluctuation can be easily handled by routing protocols because these fluctuations are predictable, unexpected sudden large change such as the flash crowd [1] often causes congestion and failure of reliable traffic control. For example, Football Association World Cup held in 1998. The web site of World Cup received 10 times larger requests than usual during the period [2]. In order to keep communication quality on the network, therefore, routing protocols that can adapt to these rapid and large traffic variations are much expected. So far, several methods to alleviate flash crowd have been proposed [3, 4]. Most of these approaches work on application level or using flow control and solution based on routing has not been studied so much.

For adaptation to environmental change in large scale networks, distributed routing protocol is more suitable than centralized protocols. Centralized routing algorithms that use global information of a network, such as OSPF[5], now starts to face difficulties in feasible operation due to significantly increasing computational costs and communication overheads accompanied with rapid growth of information networks today. In order to suppress communication overhead and realize feasible routings, distributed algorithms in which operation at each node requires only local information of a network attend much attention recently.

In order to realize distributed and self-adaptive routing protocols, various approaches that inspired by adaptive, scalable, and distributed self-organization nature of biological systems have been proposed. Biologically-inspired approaches for distributed routing protocols include AntNet[6], AntHocNet[7], where distributed agents set up efficient paths owing to innate randomness of them and indirect interaction mediated by routers. The routing protocol based on attractor selection model[8] realize adaptive path selection according to environmental change by utilizing underlying mechanism of adaptation in which the E.coli adapts to different environment [9].

Distributed routing protocols select suitable routes relying only on local information of the network, which allows protocols to realize scalable and autonomous routings. However, due to the lack of global information, these protocols often cannot respond quickly to traffic changes occurs on a network if these changes occur at outside of its local scope. For example, the routing with the attractor selection measures communication delay on paths only used for current communication without knowing traffic load or channel conditions of non-used paths, which makes it highly difficult to promptly react to, for example, unexpected improvement of channel conditions of non-used paths or traffic variation on 
out of its local scope.

Here, in order to realize distributed routing protocols that combine both stable route selection and adaptation to unexpected environmental changes beyond their local scope, we propose a distributed routing protocol based on the attractor selection model. The proposed protocol has active route exploration mechanism that can adapt to rapid traffic variation by infrequently and stochastically changing their paths in order to obtain information of non-used paths. In order to suppress excess flapping due to the route exploration and to ensure stable behaviors of the model, our extended attractor selection model has a short-term memory which stores internal states of the model before active explorations. If the exploration gives unsatisfactory results, the internal state of the protocol promptly returns to the memorized state. In order to avoid serious quality deterioration due to packet loop or congestion due to the active route change, we also introduce simple loop avoidance methods to our model.

In this paper, we first explain the routing algorithm based on biologically-inspired attractor selection model in Section II, and proposed our routing protocol in Section III. Then we describe configuration of our numerical simulation in Section IV. Discussions and conclusions are given in Section V and VI.

\section{BIOLOGICALLY-INSPIRED ATTRACTOR SELECTION}

In this Section, we provide the original attractor selection model in biological context and introduce the routing protocol based on the model. Then we propose our extended attractor selection model with stochastic exploration and short-term memory.

\section{1 original attractor selection model}

The attractor selection model is nonlinear dynamical systems that describes adaptive synthesis of nutrients in E.coli depending on surrounding environments [8]. In order to compensate missing nutrients of the environment, E.coli adaptively change its gene expression of metabolic network and synthesizes required missing nutrients. It is reported that genes corresponding to synthesis of two different nutrients suppress each other and the system reaches, after a transient state, a stable fixed point, or an attractor, corresponding to synthesis of one of these nutrients.

Concentrations of mRNA for nutrients synthesis $\vec{m}=\left(m_{1}, m_{2}\right)$ develop as,

$$
\begin{aligned}
& \frac{d m_{1}}{d t}=\frac{S(\alpha)}{1+m_{2}^{2}}-D(\alpha) m_{1}+\eta_{1} \\
& \frac{d m_{2}}{d t}=\frac{S(\alpha)}{1+m_{2}^{1}}-D(\alpha) m_{2}+\eta_{2}
\end{aligned}
$$

where $S(\alpha)=\frac{6 \alpha}{2+\alpha}$ and $D(\alpha)=\alpha$ are rate coefficients of nutrients synthesis and degradation respectively, $\alpha$ is the activity representing cell growth, $0 \leq \alpha \leq 1, \vec{\eta}=\left(\eta_{1}, \eta_{2}\right)$ are the independent white Gaussian noise corresponding to external or internal fluctuation of the gene expression.
There are two attractor $\vec{m}=\left(m, \frac{1}{m}\right)$ and $\left(\frac{1}{m}, m\right)$ satisfy $\frac{d \vec{m}}{d t}=0$ excepting the noise term. The System selects one of these two attractors according to the activity $\alpha$. When the value of the activity is low, the effect of the noise term is dominant, which makes the system fluctuate or start to random walk. If the system reaches preferable attractor due to the random walk, the value of the activity increases and fluctuation relatively decreases, which allows the system to stay the novel preferable attractor.

\section{2 routing algorithm based on the attractor selection model}

In order to apply the attractor selection model to a packet routing, the model was extended to a high dimensional system [9]. A M-dimensional variable $\vec{m}=\left(m_{1}, m_{2}, \cdots, m_{M}\right)$ develops as,

$$
\frac{d m_{i}}{d t}=\frac{s(\alpha)}{1+\left(\max m_{i}\right)^{2}-m_{i}^{2}}-d(\alpha) m_{i}+\eta_{i}
$$

where $M$ is the number of candidate nodes for next hop. $S(\alpha)=\alpha\left(b \alpha+\varphi^{*}\right), d(\alpha)=\alpha$, and $\vec{\eta}$ is $M$-dimensional independent Gaussian noise. Equation.(3) has $M$ attractors of the form as $\vec{m}=(L, L, \cdots, H, \cdots, L)$, where only $m_{k}(k=$ $1, \cdots, M)$ takes a high value while the others take a low value. When the solution of Eq.(3) is in the $k$ th attractor, the node forwards packets to the $k$ th next hop node.

Activity $\alpha$ which represents goodness of the current path at time $t$ is defined as,

$$
\alpha(t)=\frac{\min _{t-T_{w}<s \leq t} l(s)}{l(t)}
$$

where $l(s)$ is a metric, for example throughput or round trip time, of selected $k$ th path or next hop node at time $s$. In this paper, we use one-way delay as the metric $l$. In order to achieve better performance in packet routing, the system tries to converge on an attractor with higher activity or smaller delay. Each node independently calculates $\vec{m}$ and $\alpha$, and decides their next hop node in a distributed manner.

The activity is calculated from the delay of only actually selected path during past $T_{w}$ steps. Therefore, if the same attractor continues to be selected over the past time window $T_{w}$ steps, the value of the activity becomes to, which makes the system to stick to the current attractor without getting information of other paths. Because of convergence to a local solution and lack of global information, it is difficult to achieve sufficient adaptive control.

\section{3 attractor selection model with active ex- ploration}

In order to solve the problem of the routing based on existing attractor selection model, we increase variation of the noise of Eq.(3) to force the system actively exit from attractors and obtain, or search, information of non-used paths. However, stronger noise disturbs convergence of the system to 
even desirable attractors and makes whole protocol highly unstable. In order to compensate degraded stability, we introduce a short-term memory to the attractor selection model in order to stabilize the model even when it works under a stronger noise. If the system reaches an attractor with lower condition, or activity, by the stochastic active exploration, it return to a memorized state, or an attractor which the system stayed before the exploration while it moves to the new attractor if the new state gives higher activity than before.

The proposed model is given as,

$$
\begin{array}{r}
\frac{d m_{i}}{d t}=\frac{s(\alpha)}{1+\left(\max m_{i}\right)^{2}-m_{i}^{2}}-d(\alpha) m_{i}+\sigma \eta_{i} \\
-K(1-\alpha)\left(m_{i}-x_{i}\right)
\end{array}
$$

where $K$ is the constant characterizing strength of the memory term, $\tau_{\sigma}$ is the rate constant, $x_{i}$ represents the memory for $m_{i}$. The noise strength $\sigma$ is also a variable in our protocol that increases according to the following equation while the system continues to stay on an attractor.

$$
\frac{d \sigma}{d t}=-\frac{1}{\tau_{\sigma}} \sigma\left(\sigma-\sigma^{*}\right)
$$

which allows the system fluctuate and change attractor or selected path easily even if the activity remains high. We call this temporal change of attractor "exploration". In order to suppress excess instability or ensure a stable transition after route exploration, we force the value of noise strength $\sigma$ to $\delta(\fallingdotseq 0)$ just after explorations and recover it up to a constant strength $\sigma^{*}$ again according to Eq.(6).

The memory of $\vec{m}$ is denoted as $\vec{x}$ that develops as,

$$
\frac{d x_{i}}{d t}=-\frac{1}{\tau_{x}}\left(x_{i}-m_{i}\right)
$$

Similar to the forgetting curve of human's short-term memory[10], we use the exponential dependency of $x_{i}$ on $m_{i}$. The fourth term on the right side of the Eq. (5) represents attracting force from the memory state. The term is small when the system stays at good state, or the activity is high. However, the effect of memory becomes negligibly large when the system reaches a poor attractor, $\alpha<1$, due to active explorations. Then the system is pulled back to the memory state by the attraction force.

As with the traditional routing based on attractor selection, activity $\alpha$ id defined as a ratio between metric $l$ of the current and past attractors as,

$$
\begin{aligned}
\alpha(t) & =\min \left(\left(\frac{\beta}{l(t)}\right)^{n}, 1\right) \\
\frac{d \beta}{d t} & =-\frac{1}{\tau_{\beta}}(\beta-l(t))
\end{aligned}
$$

where $\beta$ is the memory of measured metrics, Because time evolution of the noise strength and memories, Eq.(6), (7), and (9) obey similar linear equation with different rate constants, we can easily adjust these constants by considering balance between frequency of exploration and speed of convergence to memory states.

\section{ROUTING PROTOCOL BASED ON THE PROPOSED MODEL}

In this section, in order to apply the proposed model to the path selection, we explain three things that we have not yet described, implementation of the model to each node, measurement of metric, or communication delay, and loop avoidance.

Each node has dynamics of the attractor selection corresponding to packet destination for each. Therefore, in the network consisting $N$ nodes, there are $N(N-1)$ attractor selection models are running on the whole network principally. Each node forwards packets based on a routing table that has pairs of destination and next hop decided by the attractor selection for the destination. Algorithm 1 describes how to update routing table on a node $P$ to a node $Q$. Each node sends Hello packet to adjacent nodes per hello interval time to confirm existing connections. If the destination of a packet is an adjacent node, the packet is directly forwarded to the router. Since route selection is not necessary for adjacent nodes, each node which has $r$ adjacent nodes runs $N-r$ dynamics of attractor selection in fact.

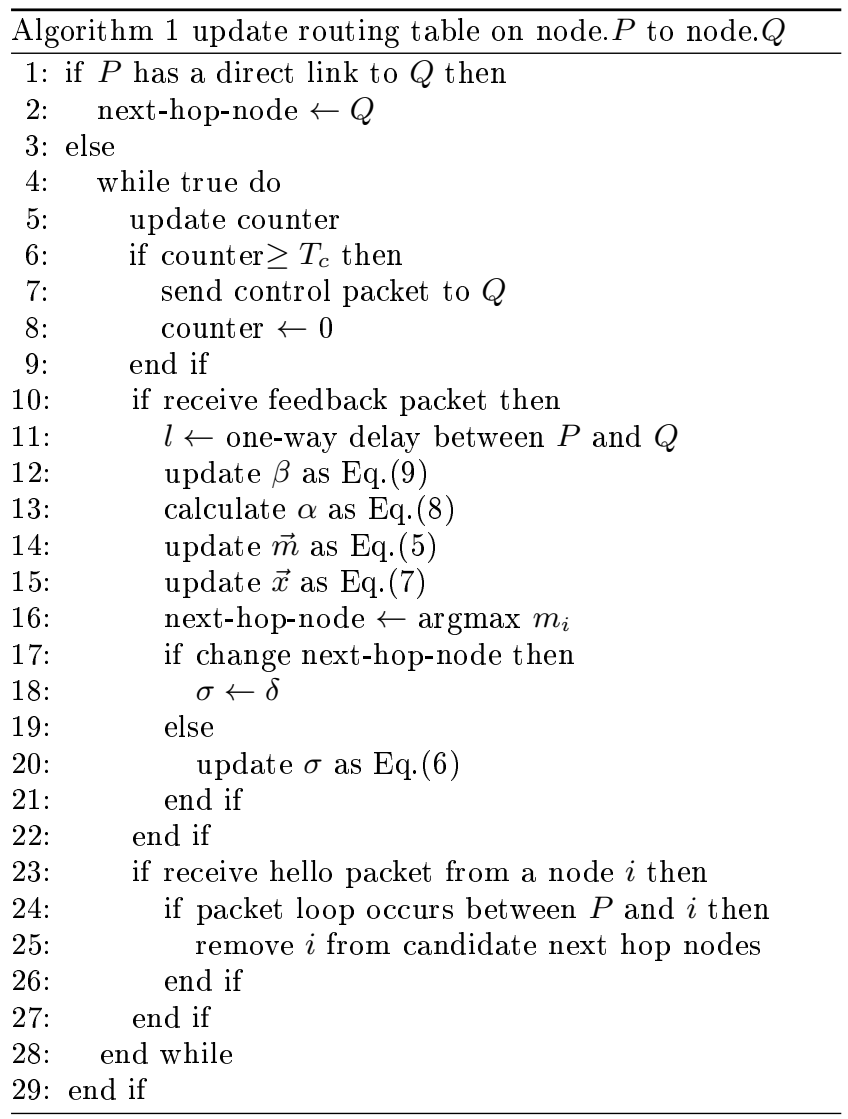

Metric $l$, or one-way delay from a source node to a destina- 
tion node is calculated as the sum of communication delay including transmission delay, queuing delay, and propagation delay. In order to measure the one-way delay along a path, each node sends control packets to destinations per control period $T_{c}$. A node that receives the control packet derive the delay by subtracting generating time of the control packet from current time, and returns a feedback packet the source node. When feedback packet reaches the source node, variables of attractor selection model such as state values $\vec{m}$ and activity $\alpha$ are updated. If the source node cannot receive feedback packet because of packet lost, $\alpha$ decreases by half without updating metric $l$.

If packets start to loop among several nodes, it may result in serious degradation of performance of routing protocols. To avoid loop as simply as possible, we introduce two loop avoidance mechanisms into the proposed protocol. First, in order to avoid loop between adjacent nodes, we make each node remove adjacent nodes who sends packets to themselves from candidate next hop nodes of route selection, which is easily realized by using hello packets that have pairs of destination node and next hop node in routeing table (line 23 to 27 of the Algorithm 1). Second, in order to reduce influence of loop consisting more than three nodes, we make nodes send a packet to a random next hop node if the packets has passed before based on its routing table.

\section{EVALUATION WITH A NETWORK SIM- ULATION}

In this section, we first explain our network simulation environments and then provide our main results with comparison to several existing methods.

\section{1 simulation environment}

We evaluate the proposed protocol in routing on a queuing network. The network is a connected random graph consisting $N=36$ nodes which is generated by the Waxman model[11]. Each pair of adjacent node is connected by $1 \mathrm{Mbps}$ bidirectional link and packet takes $1 \mathrm{~ms}$ fixed propagation delay to pass the link. Each link has buffer with size of 100Kbyte. Packets are discarded when their hop count reaches to $\mathrm{TTL}=10$ hops or queue length exceeds the buffer size. Each node generates new packet at a given packet generation rate. The packet size is distributed from 64 to 1500 Bytes based on the exponential distribution. In this simulation, we regard metric $l$ as average of communication delay of all data packets and do not model the control packet explicitly. Control period $T_{c}=100 \mathrm{~s}$.

We define values of parameters as following. Time constants of memories and noise are $\tau_{x}=0.4, \tau_{\beta}=0.2$, and $\tau_{\sigma}=5.0$. $\tau_{\sigma}$ should sufficiently larger than other two value because values of memory must converge to current state before next active route explorations. We use $\varphi^{*}=1 / \sqrt{2}, b=10$, and $\gamma=3$ based on traditional report[9]. Other values of attractor selection model are $\sigma^{*}=15, K=5, n=3$, and $\delta=0.1$ as default parameter.

\section{2 results of the network simulation}

We show adaptation of the proposed model to rapid traffic variation. Each node generates packets to all other nodes in $10 \mathrm{Kbps}$ packet generation rate. In the middle of the simula-

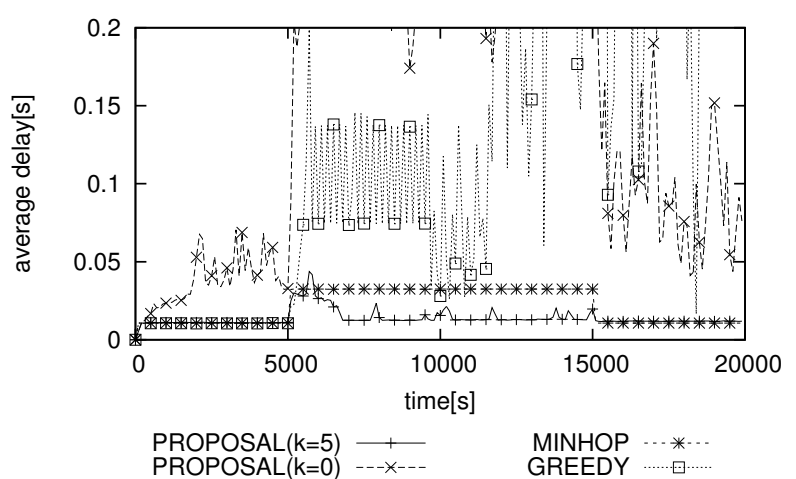

(a) average delay in download scenario

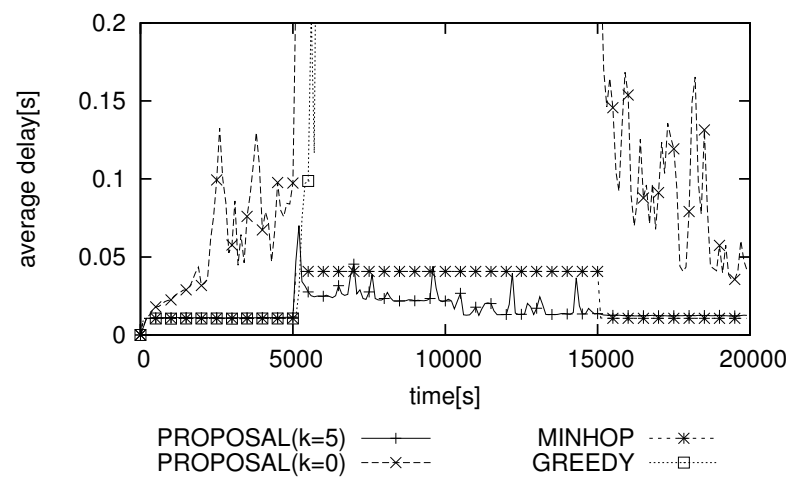

(b) average delay in upload scenario

Figure 1: time courses of average delay

tion $t=5000$, the certain node increases packet generation rates by ten, and returns to the origin rate at $t=15000$. We study two scenarios of the traffic variation; one is the download scenario in which a node increases traffic to all other nodes and another is the upload scenario in which opposite traffic, or traffic to a node from all other nodes, increases. Figure.1 shows the average delay over all simulation of the proposed model comparing to MINHOP that always selects the shortest path, and GREEDY in which each node forwards packets to a path with minimum delay at each period. $\operatorname{PROPOSAL}(k=0)$ explores non-used path owning to strengthen noise but doesn't have memory or attracting force to a memorized state.

Figure.1(a) shows average delays in the download scenario. Until time 5000 three methods other than $\operatorname{PROPOSAL}(k=$ $0)$ achieve similar better performance. When traffic variation occurs, the proposed protocol starts to change their routing table by stochastic and active exploration and each node tries to find better attractor in autonomous distributed manner, which finally make the system converge to the solution that gives smaller delay. MINHOP that continues to select the same shortest path converges to state that finally increase average delay. In GREEDY method that each node changes route selfish independently, delay is also relatively large because it leads the system to continuing flapping. Figure.1(b) shows the upload scenario. We can see that the basic tendency of the results is similar with download scenario while the proposed model takes longer time to 


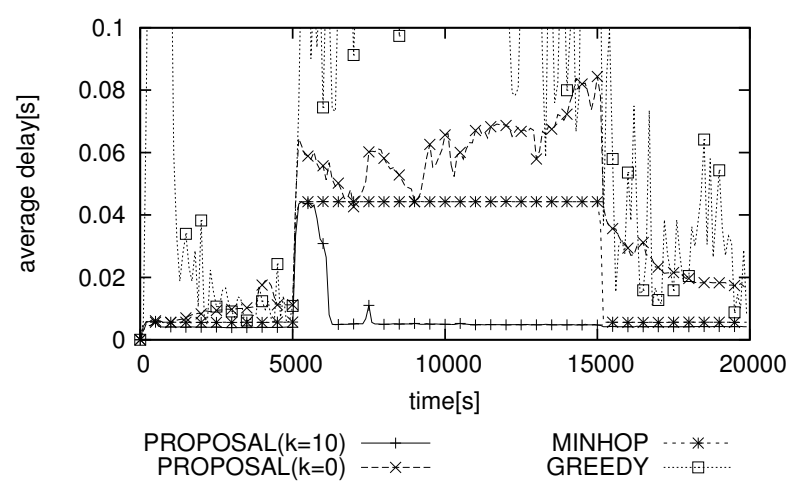

(a) average delay in download scenario

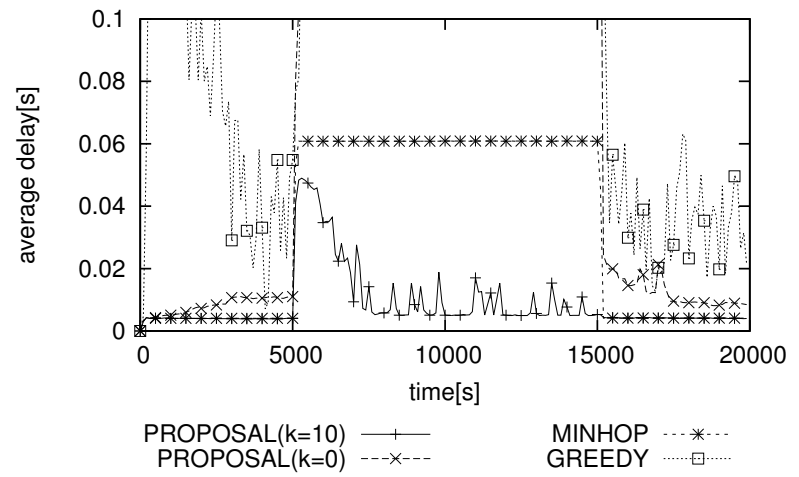

(b) average delay in upload scenario

Figure 2: time courses of average delay

reach convergence in the upload scenario and overall average delay is a litter larger than the download scenario. This difference is explained as packets from many other nodes that are concentrating on around the destination node in the upload scenario. Due to the concentration, transient rapid increase of average delay occurs in the upload scenario. We will discuss this in Section V.

Figure.2 shows results of similar evaluation on a large network consisting $N=128$ nodes, where available bandwidth of all links are adjusted to $10 \mathrm{Mbps}$ and each node generates packets in $100 \mathrm{Kbps}$. Each method perform similarly with the results of $N=36$, which may suggests that the proposed protocol is scalable for network size. Systematic evaluation of performance of the proposed protocol for ranges of size is an important future subject.

Figure. 3 shows the average delay for different values of $K$ in the download scenario. The average delay takes smaller value over a wide range excepting $K=0$. This implies that fine tuning of the model parameter $K$ is not necessary. When $K=0$, due to stochastic and active exploration, the system cannot converge to even desirable attractor and starts to random walk. The result shows that finite values of $K$ is efficiently suppress this possible instability of the proposed protocol.

Figure. 4 shows average delay for values of noise parameters $\sigma^{*}$ and $\tau_{\sigma}$ in the scenario with and without traffic varia-

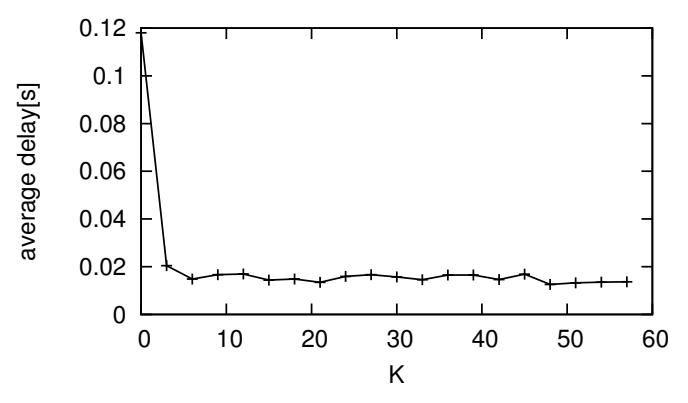

Figure 3: the evaluation of the average delay for values of K

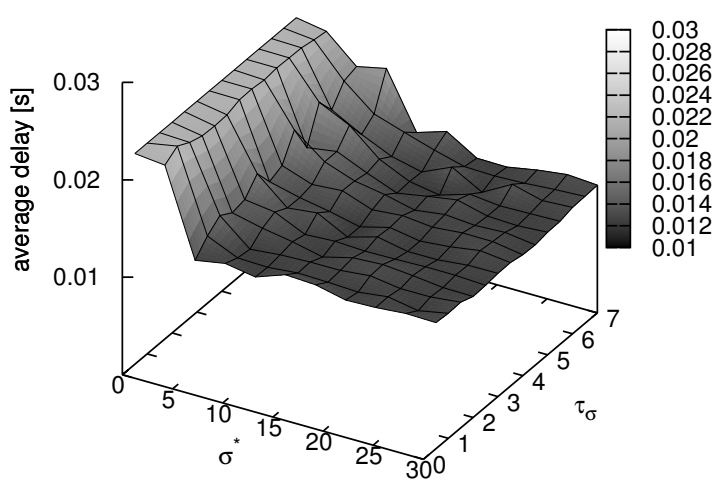

(a) average delay in download scenario

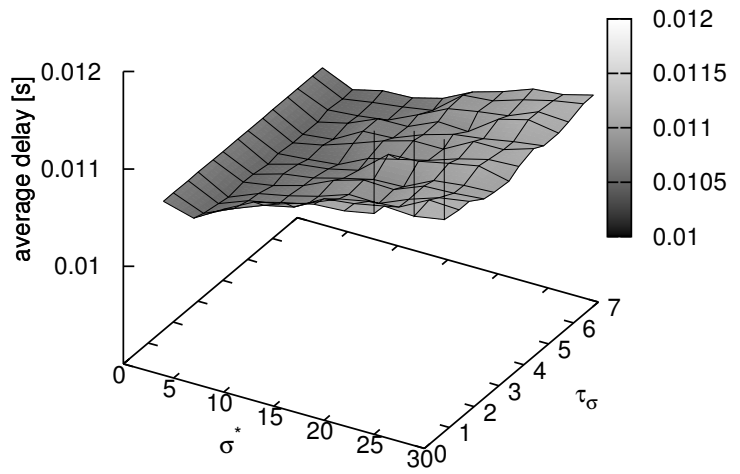

(b) average delay in normal scenario

Figure 4: the evaluation of average delay for values of $\sigma^{*}$ and $\tau_{\sigma}$

tion;the download scenario and a stationary scenario where packet generation rate is fixed to $10 \mathrm{Kbps}$ for all sourcedestination pairs. For large maximum noise strength $\sigma^{*}$ 
or small rate constant $\tau_{\sigma}$, noise strength becomes large and intervals between active route change become narrow. This frequent stochastic exploration allows the system to adapt to traffic variation more rapidly and decrease average delay as shown in the donwload scenario. Figure.4(a). However, excessive stochastic exploration decreases total performance of the protocol even if the memory term tries to stabilize the system especially for stationary scenario. In the stationary scenario Fig.4(b), though the shortest path is optimal, the proposed protocol sometimes tries to use detour route for exploration and decreases performance a little. Therefore, it is important to choose the noise parameters considering tradeoff between performance at stationary traffic and adaptation to traffic variation.

\section{DISCUSSION}

The proposed protocol can robustly responds to traffic variation and achieves higher performance, or short average delay, when large and rapid traffic changes occurs. However the protocol still sometimes leads transient increase of communication delay as shown in small spikes in Fig.1(b). In order to further improve performance of the protocol, it may be important to suppress or decrease such a transient increase of communication delay. The cause of this increase of delay is temporal congestion along a path or buffer overflow on a router induced by stochastic route change for route exploration. If a node changes its next hop node stochastically to use a link whose utilization is already high or a node that already relays many packets, inflow to the next node exceeds bandwidth and leads rapid degeneration of performance i.e. sudden increase of communication delay along the node. While the result of exploration is immediately discarded and the next hop node is returned to the memorized state in the next control period immediately, communication that shares this link can be affected. It must be an important future subject to prevent route changes that can lead such sudden congestion by utilizing additional metric such as, for example, link utilization.

\section{CONCLUSION}

In this paper, we proposed a distributed routing protocol based on the attractor selection that can adapt to rapid and large traffic variation without constantly referring global information of networks. Our protocol tries to search better solutions, or routes, over its local scope by using enhanced noise term. Simultaneously, potential instability of the routing protocol due to the active exploration is safely suppressed because the proposed system holds their state variables into their memory variables to rapidly return to the original state if results of explorations are worse. We showed validity of the proposed protocol in various scenarios of traffic change and study suitable parameter settings of the model.

\section{ACKNOWLEDGEMENT}

This work was partially supported by the Ministry of Internal Affairs and Communications with a contract entitled "R\&D for fundamental technology for energy-saving network control compatible to changing communication status" in FY2015.

\section{REFERENCES}

[1] Ari, Ismail, et al. "Modeling, analysis and simulation of flash crowds on the Internet." Storage Systems Research Center Jack Baskin School of Engineering University of California, Santa Cruz Santa Cruz, CA, Tech. Rep. UCSC-CRL-03-15 (2004).

[2] Arlitt, Martin, and Tai Jin. "A workload characterization study of the 1998 world cup web site." Network, IEEE 14.3 (2000): 30-37.

[3] Jung, Jaeyeon, Balachander Krishnamurthy, and Michael Rabinovich. "Flash crowds and denial of service attacks: Characterization and implications for CDNs and web sites." Proceedings of the 11th international conference on World Wide Web. ACM, 2002.

[4] Yu, Shui, et al. "Discriminating DDoS attacks from flash crowds using flow correlation coefficient." Parallel and Distributed Systems, IEEE Transactions on 23.6 (2012): 1073-1080.

[5] R. Coltun, D. Fergunson and J. Moy, "OSPF for IPv6", RFC 2740, pp. 1-94, Dec. 1999.

[6] Di Caro, Gianni, and Marco Dorigo. "AntNet: Distributed stigmergetic control for communications networks." VIVEK-BOMBAY- 12.3/4 (1999): 2-37.

[7] Di Caro, Gianni, Frederick Ducatelle, and Luca Maria Gambardella. "AntHocNet: an adaptive nature]inspired algorithm for routing in mobile ad hoc networks." European Transactions on Telecommunications 16.5 (2005): 443-455.

[8] A.Kashiwagi, I.Urabe, K.Kaneko, and T.Yomo, "Adaptive response of a gene net-work to environmental changes by fitness-induced attractor selection", PLoS ONE, 1(1):e49(1-10),Dec.2006.

[9] N. Onzuka, N. Wakamiya, and M. Murata, "Robust and lightweight routing with attractor selection", in Proceedings of World Conference on Information Technology (WCIT 2013), Dec. 2013.

[10] London, I.D. 1950, âĂIJAn ideal equation derived for a class of forgetting curvesâĂ 57, 295-302

[11] Waxman, Bernard M. "Routing of multipoint connections." Selected Areas in Communications, IEEE Journal on 6.9 (1988): 1617-1622. 